\title{
A Computational Approach towards Just Intonation for Renaissance Music
}

\author{
Kevin K.H. Cheung \\ School of Mathematics and Statistics, Carleton University, Ottawa, ON K1S 5B6, Canada \\ *Corresponding Author: kevin.cheung@carleton.ca
}

Copyright (C) 2013 Horizon Research Publishing All rights reserved.

\begin{abstract}
A computational realization of Ross W. Duffin's approach towards obtaining just tuning for Renaissance music is described. The method is implemented in Optune, a tool developed in Python.
\end{abstract}

Keywords Tuning, Temperament, Music, Just Intonation, Renaissance, Computation, Integer Programming

\section{Introduction}

Much of Western music is based on the 12-note octave. At present, the most common tuning is equal temperament (ET) in which every semitone has the same ratio $2^{1 / 12}$. However, for performances of early music, historical tunings are often used instead. Under these tunings, not all semitones have the same ratio. Different tunings favour the acoustic purity of intervals differently. Just tuning (also known as Ptolemaic tuning) is one that uses simple ratios for intervals. The $3 \mathrm{rds}$, 4ths, 5ths, and 6ths under just tuning are regarded as acoustically pure as a result of the absence of beats.

With keyboard and fretted instruments such as the piano, harpsichord, guitar etc., the choice of which tuning to use depends on the work to be performed and must be decided ahead of time. (It should be mentioned that such a limitation can be overcome by technology such as Pitch Palette [1] for an electronic keyboard.) For non-fixed-pitch instruments such as human voices and some strings and wind instruments, various degrees of flexibility exist in the choice of pitches at any given time. Hence, it is possible to dynamically change the tuning of the notes as one progresses in the performance of a piece to favour acoustically pure intervals.

The question of when and how much to adjust the pitches is one of the many aspects of music interpretation. One ideal is to use just intervals throughout. However, it is known that doing so could lead to problems. (See Section 2.) Such problems are especially noted by Blackwood [2] who concluded that "just tuning is of no practical use with regard to the existing Western repertoire" (p.153).

Yet it is difficult deny that just intervals have a certain appealing quality (see [3] for audio samples) as evidenced by performances by early music choral ensembles. Duffin [4] demonstrated that just tuning could be adopted in a satisfactory manner provided one accepts occasional unjust intervals. He argued that the sacrifice that one has to make in this regard could be small when it comes to Renaissance music.

Duffin's approach could be viewed as one of minimizing a penalty measure on unjustness. Even though Duffin gave neither an explicit definition of the measure nor a mechanical procedure in obtaining the final choices of pitches, one can infer from his work that minimizing the occurrences of unjust intervals is the goal, taking musical sensibilities into account. Given this understanding and possibly ignoring the subjective aspect of aesthetics, we show how one can formulate the problem of determining the tuning of notes throughout a piece as an integer linear programming problem. The contribution of this paper is a general description of the approach as implemented in Optune [5], a tool written in Python. It uses open-source packages Music21 [6] and PyMathProg [7] and produces Csound [8] files from Music21 scores. Previous work by Polansky et al. [9] applied optimization techniques to obtaining optimal temperament for fixed-pitch instruments.

The rest of the paper is organized as follows: Section 2 outlines the problems of using just tuning exclusively and Duffin's approach to these problems. Section 3 describes the implementation details in one realization of Duffin's approach. Section 4 discusses some results obtained from applying Optune though no attempt is made to justify the musical merits of the results. A forthcoming companion paper will assess the impact of optimally tuned music on listeners, an aspect that is beyond the scope of the present paper.

The reader is assumed to have a working knowledge of basic music theory and the mathematics of tuning. (See [3] for a quick guide and audio examples.)

To facilitate discussion, we adopt two conventions. The first is that when we speak of an interval in the context of being just or unjust, it is a $3 \mathrm{rd}, 4 \mathrm{th}, 5 \mathrm{th}, 6 \mathrm{th}$, unison, or their octave equivalents. Just 2 nds and 7 ths certainly exist but they are not taken into account as they are not a basis for forming triads or their inversions. The second convention is that, 
unless otherwise stated, notes having the same note name but at different octaves are considered to be the same when it comes to tuning. Hence, the ratios for the notes are always at least 1 and less than 2 .

\section{Duffin's Proposal}

The diatonic scale starting on $\mathrm{C}$ has the following notes: $\mathrm{C}$, D, E, F, G, A, B. They correspond to the white keys on a standard musical keyboard.

The ratios, relative to the starting $\mathrm{C}$, for Pythagorean tuning and just tuning are:

Table 1. Ratios for Pythagorean and just tuning

\begin{tabular}{|c|c|c|}
\hline Note & Pythagorean & Just \\
\hline C & 1 & 1 \\
\hline D & $9 / 8$ & $10 / 9$ \\
\hline E & $81 / 64$ & $5 / 4$ \\
\hline F & $4 / 3$ & $4 / 3$ \\
\hline G & $3 / 2$ & $3 / 2$ \\
\hline A & $27 / 16$ & $5 / 3$ \\
\hline B & $243 / 128$ & $15 / 8$ \\
\hline
\end{tabular}

Under Pythagorean tuning, 4ths and 5ths are acoustically pure with ratios $4 / 3$ and $3 / 2$, respectively. But 3rds and 6ths sound rather harsh as a result of complex ratios. For example, the major 3rds C-E, F-A, and G-B have ratios 81/64 whereas the corresponding ratios under just tuning are $5 / 4$. In general, $5 / 4$ is the ratio for a just major $3 \mathrm{rd}, 6 / 5$ for a just minor $3 \mathrm{rd}$, $8 / 5$ for a just minor 6 th, and $5 / 3$ for a just major 6 th. As a result, if a piece of music contains many 3rds (as well as 6 ths), playing the notes using Pythagorean tuning can result in a performance with a perceptible harshness. (It should be pointed out that the 5 th given by $\mathrm{D}-\mathrm{A}$ in the just tuning of the diatonic scale as shown above is nowhere near pure and is a well-known undesirable trait of the tuning.)

One might wonder if it is at all possible to use just intervals throughout a piece. Consider the example as shown in Figure 1.

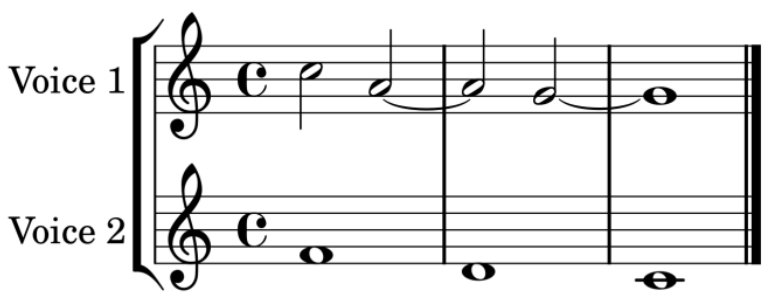

Figure 1. Impossible to have just intervals throughout

Suppose that all the intervals are just. Expressing the pitches in terms of ratios relative to the middle $\mathrm{C}$ with value 1 , the beginning $\mathrm{C}$ in Voice 1 has value 2 and the beginning $\mathrm{F}$ in Voice 2 being a perfect 5 th below has value $4 / 3$. The $A$ in Voice 1 is a major $3 \mathrm{rd}$ above this $\mathrm{F}$ and so has value $5 / 3$. The $\mathrm{D}$ in Voice 2 is a perfect 5 th below the $\mathrm{A}$ and so has value $10 / 9$. The $\mathrm{G}$ in Voice 1 is a perfect 5 th above the $\mathrm{D}$ and so has value 40/27. The final $\mathrm{C}$ in Voice 2 is a 5 th down and so has value $80 / 81$, which is less than 1 , the value for the middle $C$ !

Hence, maintaining just intervals throughout this example leads to pitch drift. As a corollary, if one insists that the value of the final $\mathrm{C}$ be 1 , it is impossible to have all intervals to be just.

From this simple, albeit contrived, example, the inherent difficulty in adopting just tuning in Western music is apparent. (More examples of problematic passages in existing Renaissance repertory are considered by Blackwood [2].) One obvious solution to such a problematic passage is to use a tempered tuning. Another solution is to admit occasional unjust intervals. Duffin [4] would permit the interval F-A or D-A to be unjust: One could use Pythagorean tuning for the example so that all the 5ths are pure but sacrificing the purity of the major 3rd F-A. Or instead, one could maintain purity of F-A and sacrifice the purity of D-A by setting $D$ to $9 / 8$. It is perhaps debatable if either of these solutions is more appealing to listeners than one that uses a tempered tuning. However, it is not the purpose of this paper to discuss the artistic merits of Duffin's approach. The objective is to describe a mechanized decision process on where to place the unjust intervals as we will do next.

\section{Realization}

As can be seen in [4], one can choose from a list of more than two pitches for each note. However, for simplicity, we begin by having at most two pitches per note. Later, we describe a slight extension to more than two pitches per note for some notes that seem to work better for pieces with higher complexity. Our Python tool Optune is built in such a way that accommodates an arbitrary number of pitches per note.

We will be working in the key of $\mathrm{C}$ (or its relative minor, A minor.) If a piece is in a different key, it is first transposed to $\mathrm{C}$, then analyzed and then transposed back. It is also assumed that there are no $\mathrm{E} \#, \mathrm{~B} \#, \mathrm{C} \mathrm{b}, \mathrm{Fb}$, and no note with two or more sharps or flats. This limitation is not at all crippling in practice since Renaissance music rarely modulates too far from the home key as evidenced by the commonly performed pieces today. As a start, we include the ratios from both Pythagorean and just tunings for the notes $C, D, E, F, G, A, B$. The ratios for $F \#, C \#$, $\mathrm{G} \#$, and $\mathrm{D} \#$ are taken from just major 3rds above the ratios for D, A, E, and B, respectively. Finally, the ratios for $\mathrm{A} \#$ are taken from just major 3rds above the ratios for $\mathrm{F} \#$. Table 2 summarizes the ratios for these notes.

The ratios for $\mathrm{B} b$ are taken from just minor 6ths above the ratios for $\mathrm{D}$. The ratios for $\mathrm{E} b$ are taken from perfect 
5 ths below the ratios for $\mathrm{B} b$. The ratios for $\mathrm{A} b$ are taken from perfect 4 ths above the ratios for $E b$. The ratios for $\mathrm{D} b$ are taken from perfect 5 ths below the ratios for $\mathrm{A} b$. Finally, the ratios for $G b$ are taken from just major 3rds below the ratios for $\mathrm{B} b$. Table 3 summarizes the ratios for these notes.

Table 2. Ratios for sharps

\begin{tabular}{|c|c|}
\hline Note & Ratios \\
\hline C\# & $25 / 24,135 / 128$ \\
\hline D\# & $75 / 64,1215 / 1024$ \\
\hline F\# & $25 / 18,45 / 32$ \\
\hline G\# & $25 / 16,405 / 256$ \\
\hline A\# & $125 / 72,225 / 128$ \\
\hline
\end{tabular}

Table 3. Ratios for flats

\begin{tabular}{|c|c|}
\hline Note & Ratios \\
\hline $\mathrm{D} b$ & $16 / 15,256 / 243$ \\
\hline $\mathrm{E} b$ & $32 / 27,6 / 5$ \\
\hline $\mathrm{G} b$ & $64 / 45,36 / 25$ \\
\hline $\mathrm{A} b$ & $8 / 5,128 / 81$ \\
\hline $\mathrm{B} b$ & $125 / 72,225 / 128$ \\
\hline
\end{tabular}

The above choices of ratios are to ensure that there is at least one just major triad and at least one just minor triad starting on each note. We now describe an integer linear programming model for deciding which pitch to use for each note so that the occurrences of unjust 3rds, 4ths, 5ths, 6 ths and unisons are minimized. Instead of specifying the variables and constraints in a set-theoretic language, we simply illustrate the construction by examples.

First of all, it is assumed that each part is monophonic. For each note in each part, whether or not it is a tied note, a binary variable is created for each of the possible ratios. In the example above, the first measure will give four variables, one for $\mathrm{C}$ (call it $x_{1, \mathrm{C}}$ where 1 in the subscript indicates the part), two for A (call them $x_{1, \mathrm{~A} 5 / 3}$ and $x_{1, \mathrm{~A} 27 / 16}$ ), and one for $\mathrm{F}$ (call it $x_{2, \mathrm{~F}}$ ). As exactly one pitch per note must be chosen, we need to add a constraint that says the sum of all the variables for each note is 1 . Hence, the $\mathrm{A}$ in the first measure in part 1 gives the constraint $x_{1, \mathrm{~A} 5 / 3}+x_{1, \mathrm{~A} 27 / 16}=1$ and the $\mathrm{F}$ in part 2 gives the constraint $x_{2, \mathrm{~F}}=1$.

Note that at the beginning of the second measure, there is a tied $\mathrm{A}$ in part 1. If the variables for this $\mathrm{A}$ are $x_{2, \mathrm{~A} 5 / 3}$ and $x_{2, \mathrm{~A}_{27 / 16}}$, we add the constraints $x_{1, \mathrm{~A}_{5 / 3}}=x_{2, \mathrm{~A}_{5 / 3}}$ and $x_{1, \mathrm{~A}_{27 / 16}}=$ $x_{2, \mathrm{~A}_{27} 716}$ to ensure that tied notes receive the same pitches. All other tied notes are handled similarly. We also impose such constraints on consecutive notes having the same note name that are no more than a measure apart since differing pitches for such notes are perceived as being out of tune. (See [4] for a discussion.)

If two notes from different parts forming a 3rd, 4th, 5th, 6th, or unison overlap in time, a binary variable is created for each ratio pair that does not give a just interval. From the first measure, we create the variable $z_{\left(1, \mathrm{~A}_{2} 7 / 16\right),(2, \mathrm{~F})}$ and add the constraint $z_{(1, \mathrm{~A} 27 / 16),(2, \mathrm{~F})} \geq x_{1, \mathrm{~A} 27 / 16}+x_{2, \mathrm{~F}}-1$. When the variable is minimized, it will equal to the maximum of 0 and the right-hand side. Note that the right-hand side is nonzero (in fact, 1) when both $x$ variables are set to 1; i.e. when the ratios these variables are chosen, in which case, the interval F-A will not be just. Thus minimizing the variable $z_{(1, \mathrm{~A} 27 / 16),(2, \mathrm{~F})}$ tells the solver to avoid choosing unjust intervals if possible.

In our implementation, the variable $z_{\left(1, \mathrm{~A}_{27 / 16),(2, \mathrm{~F})}\right.}$ appears in the objective function with coefficient equal to the duration of overlap in terms of quarter notes multiplied by a penalty factor. The penalty factor for 3rds and 6ths is 1 , that for 4 ths and 5 ths is 5 and that for unisons is 10 . When the objective function is minimized, 4ths, 5ths, and unisons are favoured as these intervals sound jarring when mistuned.

To allow for the possibility of favouring a particular ratio when there is a choice for a note, one can add the less favoured note-ratio variable to the objective function with a relatively small penalty coefficient (e.g. 0.01). For example, if we prefer A to use the ratio 5/3 when it is not involved in an interval that is of interest, we can add the variable for the ratio $27 / 16$ with a coefficient of 0.01 , say, into the objective function. This way, when the objective function is minimized, it will favour setting the variable for the ratio $5 / 3$ to 1 .

The above completes the description of the integer linear programming model as constructed by Optune. The number of ratios per note is not at all a restriction. Optune has the option of using an extended set of ratios which include $27 / 20$ for $F, 40 / 27$ and $243 / 160$ for $G$, and 50/27 for B. (One could add 81/80 to $\mathrm{C}$ as Duffin had done but we preferred to have a stable C.) These additional ratios seem to result in more just intervals when a piece contains fragments in A minor (or A mode). 

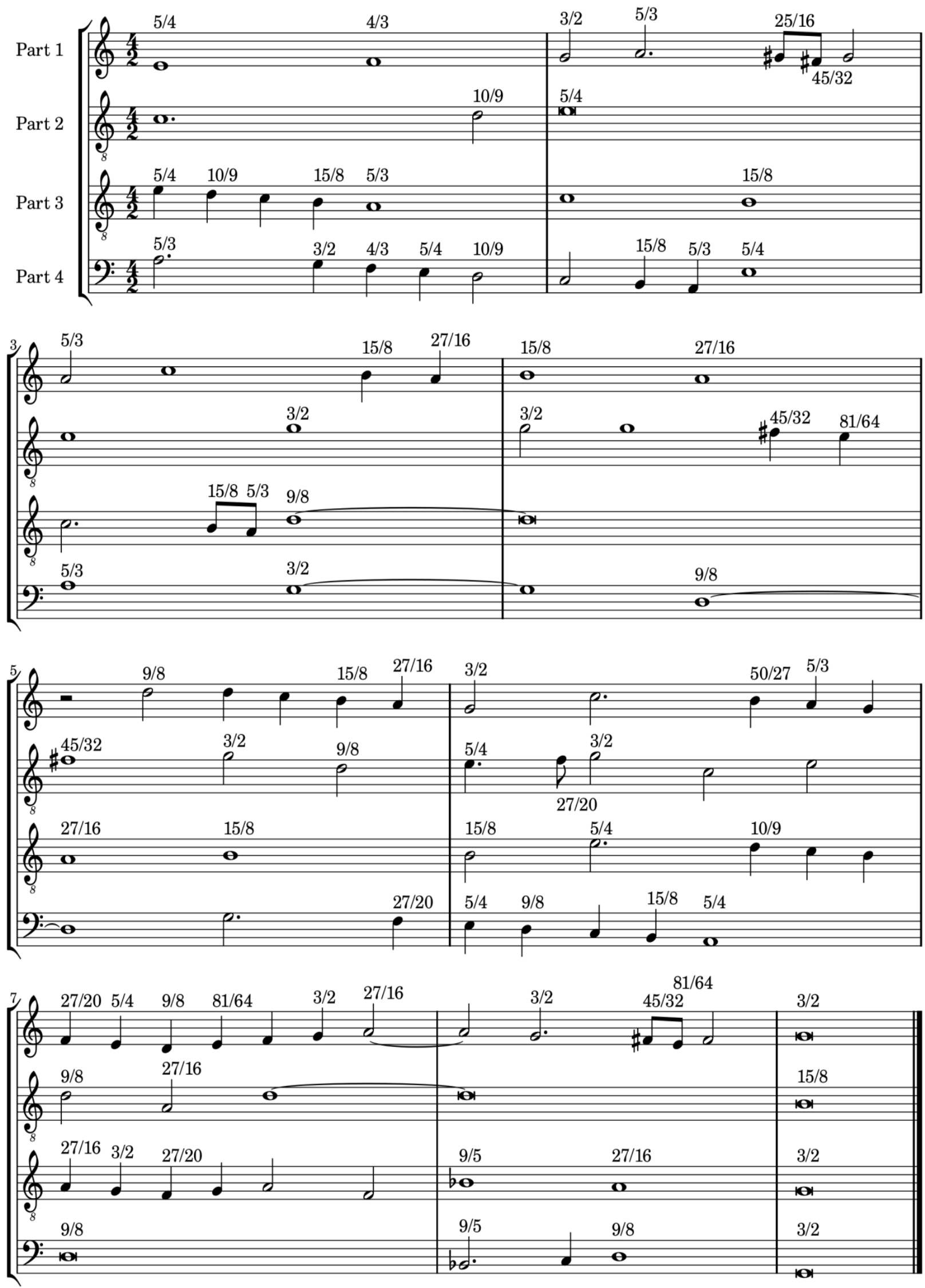

Figure 2. Optune's solution for Example 5b in [4] 


\section{Results}

Using GLPK [10] as the integer programming solver via PyMathProg, the 4-part pieces (no more than a few pages long) to which we applied Optune were tuned in a matter of seconds.

It is worth nothing that for simple or short pieces, it is entirely possible to arrive at an optimal tuning by hand. However, for pieces with complex lines, Optune provides an invaluable means to finding an optimal tuning. Even if the resulting tuning might not satisfy every musical taste, it provides a solid reference for finding an aesthetically satisfying tuning. We now illustrate the benefits of Optune by examining Example 5b in [4], one of the most complex examples considered by Duffin [4] and Blackwood [2].

The tuning given by Duffin contains three unjust intervals: $\mathrm{C}-\mathrm{G}$ in measure 3 with the $\mathrm{C}$ in part 1 at $81 / 80$ and $\mathrm{G}$ at $3 / 2$, and two occurrences of $\mathrm{G}-\mathrm{E}$ in the first half of measure 7 with $E$ in part 1 at $81 / 64$ and $G$ in part 3 at 3/2. (The first occurrence could be made just simply by setting $\mathrm{E}$ to 5/4.) Using the extended set of ratios, Optune found a tuning that has only the second occurrence of G-E in measure 7 unjust. (See Figure 2 for Optune's solution.) In fact, it can be seen that an unjust interval at that point cannot be avoided. Therefore, Optune found an optimal tuning for the piece, avoiding the unjust 5 th $\mathrm{C}-\mathrm{G}$ even though Duffin regarded it is tolerable.

We mention in passing that if the extended set of ratios had not been used, there would be a few more unjust intervals.

\section{Conclusion}

A realization for Duffin's approach towards just tuning for Renaissance music using integer linear programming is described in this paper and is implemented in Optune, a tool written in Python that generates optimally tuned performances in Csound files. The tool makes it apparent how many intervals can be made just in a piece of music with respect to a reference of permissible pitches. Before Optune, finding the desired pitches to form as many just intervals as possible in a piece had to be done manually. Early music performers now have access to a free application that automates the process.

An important question that is not addressed in this paper is whether or not such optimally tuned performances are preferable to ones under a historical temperament. This question deserves attention because ultimately a piece of music is performed and heard, not mathematically analyzed. To satisfactorily address this question will require a separate study involving human subjects.

\section{Acknowledgements}

The author would like to thank Amy Heron, Stephanie Maitland, and Joe Stalmach for their help in evaluating an early version of Optune.

\section{REFERENCES}

[1] Pitch Palette MIDI tuning software for pure harmony, Justonic Tuning Inc. Online available from http://www.justonic.com

[2] E. Blackwood, The Structure of Recognizable Diatonic Tunings, Princeton, 1985.

[3] D. Barlett, The Mathematics of Tuning and Temperament. Online available from

http://www.pyxidium.u-net.com/Acoustics/MusicMaths/Mus icMaths.html

[4] R.W. Duffin, Just Intonation in Renaissance Theory and Practice, Music Theory Online, 12(3), 2006. Online available from

http://www.mtosmt.org/issues/mto.06.12.3/mto.06.12.3.duffi n.html

[5] K.K.H. Cheung, Optune: Python tool for obtaining just tuning optimally. Online available from

http://people.math.carleton.ca/ kcheung/optune

[6] Music21: a Toolkit for Computer-Aided Musicology, version 1.5. Online available from http://web.mit.edu/music21

[7] Y. Lan, PyMathProg, version 0.4.2. Online available from http://pymprog.sourceforge.net

[8] Csound, version 5.0. Online available from http://www.csounds.com

[9] L. Polansky, D. Rockmore, M.K. Johnson, D. Repetto, W. Pan, A mathematical model for optimal tuning systems, Perspectives of New Music, 47(1), pp. 69-110, 2009.

[10] GLPK (GNU Linear Programming Kit), Online available from http://www.gnu.org/software/glpk 